\title{
RATE OF CONVERGENCE OF GUPTA-SRIVASTAVA OPERATORS BASED ON CERTAIN PARAMETERS
}

\author{
RAM PRATAP AND NAOKANT DEO
}

Abstract. In the present paper, we consider the Bézier variant of the Gupta-Srivastava operators [14] and discuss some direct convergence results by using of Lipschitz type spaces, Ditzian-Totik modulus of smoothness, weighted modulus of continuity and for functions whose derivatives are of bounded variation. In the end some graphical representation for comparison with other variants have been presented.

Mathematics subject classification (2010): 41A25, 41A36.

Keywords and phrases: Baskakov operators, hypergeometric function, Lipschitz type space, DitzianTotik modulus of smoothness, functions of bounded variation.

\section{REFERENCES}

[1] T. ACAR, L. N. Mishra And V. N. Mishra, Simultaneous approximation for generalized Srivastava-Gupta operators, J. Funct. Spaces, (2015), Article ID 936308, 1-11.

[2] P. N. Agrawal,S. Araci, M. Bohner And L. Kumari, Approximation degree of DurrmeyerBézier type operators, J. Ineql. Appl., (2018), 1-17.

[3] N. DEO, A note on equivalent theorem for Beta operators, Mediterr. j. math., 4, (2007), 245-250.

[4] N. DEO, Faster rate of convergence on Srivastava-Gupta operators, Appl. Math. Comput., 21, 218(2012), 10486-10491.

[5] M. Dhamija, R. Pratap And N. Deo, Approximation by Kantorovich form modified SzászMirakyan Operators. Appl Math Comput,317, (2018), 109-120.

[6] Z. Ditzian And V. Totik, Moduli of smoothness, Springer series in computational Mathematics, 9 , (1987), Springer New York.

[7] V. Gupta And H. M. SRivastava, A General family of the Srivastava-Gupta operators preserving linear functions, European J. Pure Appl. maths, 3, 11(2018), 575-579.

[8] N. ISPIR AND I. YÜKSEL, On the Bézier variant of Srivastava-Gupta operators, Appl. Math. E-Notes, 5, (2005), 129-137.

[9] A. KaJla, On the Bézier variant of the Srivastava-Gupta Operators, Constructive Mathematical Analysis, 1, (2018), 99-107.

[10] A. KUMAR, Approximation by Stancu type generalized Srivastava-Gupta operators based on certain parameter, Khayaam J. Math., 3, (2017), 147-159.

[11] P. MAheswari (Sharma), On modified Srivastava-Gupta operators, Filomat, 6, 29(2015), 1173 1177.

[12] T. NEER, N. ISPIR AND P. N. AgRAWAL, Bézier variant of modified Srivastava-Gupta operators, Revista de la Unio In Matema Itica Argentina, 2, 58(2017), 199-214.

[13] Özarslan M.A. and Duman O., Local approximation behaviour of modified SMK operators, Miskolk Math.Notes, 11(1)(2010), 87-99.

[14] R. Pratap And N. Deo, Approximation by Genuine Gupta-Srivastava Operators, RACSAM, (2019), https://doi.org/10.1007/s13398-019-00633-4.

[15] H. M. SRIVAstaVA AND V. GuPtA, A Certain family of summation-integral type operators, Math. Comput. Modelling, 37, (2003), 1307-1315.

[16] D. K. VERMA AND P. N. AgRAWAL, Convergence in simultaneous approximation for SrivastavaGupta operators, Math. Sci., Springer, 6, (2012), Article ID 22, 8p. 
[17] R. Yadav, Approximation by modified Srivastava-Gupta operators, Appl. Math. Comput., 226, (2014), 61-66.

[18] I. YÜKSEL AND N. ISPIR, Weighted Approximation by a certain family of Summation integral-type operators, Comput. Math. Appl., 52, (10-11)(2007), 1463-1470. 